Vol. 45 (1992) [249-260]

\title{
EXISTENCE THEOREMS FOR A MULTIVALUED BOUNDARY VALUE PROBLEM
}

\author{
Salvatore A. Marano
}

Let $F$ be a multifunction from $[a, b] \times \mathbb{R}^{n} \times \mathbb{R}^{n}$ into $\mathbb{R}^{n}$, with non-empty closed convex values. In this paper we prove that, under suitable assumptions, the multivalued boundary value problem

$$
\left\{\begin{array}{l}
u^{\prime \prime} \in F\left(t, u, u^{\prime}\right) \\
u(a)=u(b)=0
\end{array}\right.
$$

has at least one solution $u \in W^{2, p}\left([a, b], \mathbf{R}^{n}\right)$. Next we point out some particular cases.

\section{INTRODUCTION}

Let $[a, b]$ be a compact real interval with the Lebesgue measure structure; $n$ a positive integer; $\mathbb{R}^{n}$ the real Euclidean $n$-space, whose zero element is denoted by $0 ; p \in[1,+\infty] ; W^{2, p}\left([a, b], \mathbb{R}^{n}\right)$ the space of all $u \in C^{1}\left([a, b], \mathbb{R}^{n}\right)$ such that $u^{\prime}$ is absolutely continuous in $[a, b]$ and $u^{\prime \prime} \in L^{p}\left([a, b], \mathbb{R}^{n}\right) ; F$ a multifunction from $[a, b] \times \mathbb{R}^{n} \times \mathbb{R}^{n}$ into $\mathbb{R}^{n}$, with non-empty closed convex values.

Consider the problem

$$
\left\{\begin{array}{l}
u^{\prime \prime} \in F\left(t, u, u^{\prime}\right) \\
u(a)=u(b)=0
\end{array}\right.
$$

A function $u:[a, b] \rightarrow \mathbb{R}^{n}$ is said to be a generalised solution of (P) if $u \in$ $W^{2, p}\left([a, b], \mathbb{R}^{n}\right), u(a)=u(b)=0$ and, for almost every $t \in[a, b]$, one has $u^{\prime \prime}(t) \in$ $F\left(t, u(t), u^{\prime}(t)\right)$.

In this paper we prove that, under suitable assumptions, problem $(\mathrm{P})$ has at least one generalised solution (see Theorem 2.1). Further, as a simple consequence of Theorem 2.1, we obtain a result (Theorem 2.2) which improves Theorem 3 of [7] and, for $F$ single-valued and continuous, gives Theorem 4.2, p.424, of [6] (see also [1], Theorem 1.1.2). Afterwards, we point out some particular cases of Theorem 2.2.

Received 3rd April 1991

Copyright Clearance Centre, Inc. Serial-fee code: 0004-9729/92 \$A2.00+0.00. 
As far as we know, one of the most used methods to get existence results for problem (P) is that of the topological transversality by A. Granas (or similar degree-theoretic arguments). In order to apply this method, one needs conditions which guarantee a priori bounds, with respect to the norm $\max _{t \in[a, b]}\|u(t)\|_{\mathbb{R}^{n}}$ on $C^{0}\left([a, b], \mathbb{R}^{n}\right)$, for the solutions and their first order derivatives of a suitable family of problems related to $(P)$ (see, for instance, $[3,4,5]$ ). Our approach is rather different and is based on a very recent existence theorem for operator inclusions by $O$. Naselli Ricceri and $B$. Ricceri (see [7], Theorem 1).

\section{Preliminaries}

Let $A, B$ be two non-empty sets. A multifunction $\Phi: A \rightarrow 2^{B}$ is a function from $A$ into the family of all subsets of $B$. The graph of $\Phi$ is the set $\{(a, b) \in A \times B: b \in$ $\Phi(a)\}$. If $(A, \mathcal{F})$ is a measurable space and $B$ is a topological space, we say that $\Phi$ is measurable if, for every open set $\Omega \subseteq B$, the set $\{a \in A: \Phi(a) \cap \Omega \neq \phi\}$ belongs to $\mathcal{F}$.

Let $(\Sigma, \delta)$ be a metric space. For every $\sigma \in \Sigma$ and every non-empty set $\Omega \subseteq \Sigma$, put:

$$
\delta(\sigma, \Omega)=\inf _{\omega \in \Omega} \delta(\sigma, \omega)
$$

Now, let $I$ be a compact real interval and let $k \in[1,+\infty]$. We denote by $W^{2, k}\left(I, \mathbb{R}^{n}\right)$ the space of all $u \in C^{1}\left(I, \mathbb{R}^{n}\right)$ such that $u^{\prime}$ is absolutely continuous in $I$ and $u^{\prime \prime} \in L^{k}\left(I, \mathbb{R}^{n}\right)$, where $u^{\prime}=d u / d t$ and $u^{\prime \prime}=d^{2} u / d t^{2}$.

If $u \in W^{2, k}\left([a, b], \mathbb{R}^{n}\right)$ and $u(a)=u(b)=0$, then it is easy to check that for every $t \in[a, b]$ one has

$$
\begin{gathered}
u(t)=-\int_{a}^{b} G(t, \sigma) u^{\prime \prime}(\sigma) d \sigma, \quad u^{\prime}(t)=-\int_{a}^{b} \frac{\partial G(t, \sigma)}{\partial t} u^{\prime \prime}(\sigma) d \sigma, \\
G(t, \sigma)= \begin{cases}\frac{(b-t)(\sigma-a)}{b-a} & \text { for } a \leqslant \sigma \leqslant t \leqslant b \\
\frac{(b-\sigma)(t-a)}{b-a} & \text { for } a \leqslant t \leqslant \sigma \leqslant b .\end{cases}
\end{gathered}
$$

The following lemma will be useful in the sequel. 
LEMMA 1.1. Let $k \in[1,+\infty[$. Then, for every $t \in[a, b]$ one has:

$$
\begin{aligned}
\left(\int_{a}^{b}|G(t, \sigma)|^{k} d \sigma\right)^{1 / k} & \leqslant \frac{(b-a)^{1+1 / k}}{4(k+1)^{1 / k}} \\
\left(\int_{a}^{b}\left|\frac{\partial G(t, \sigma)}{\partial t}\right|^{k} d \sigma\right)^{1 / k} & \leqslant \frac{(b-a)^{1 / k}}{(k+1)^{1 / k}} \\
\sup _{\sigma \in[a, b]}|G(t, \sigma)| & \leqslant \frac{b-a}{4} ; \\
\sup _{\sigma \in[a, b] \backslash\{t\}}\left|\frac{\partial G(t, \sigma)}{\partial t}\right| & \leqslant 1 .
\end{aligned}
$$

Proof: When $k=1,\left(i_{1}\right)$ is well-known (see, for instance, [6], p.422); hence, we may suppose $k>1$. For every $t \in[a, b]$ one has

$$
\begin{aligned}
\left(\int_{a}^{b}|G(t, \sigma)|^{k} d \sigma\right)^{1 / k} & =\left[\left(\frac{b-t}{b-a}\right)^{k} \int_{a}^{t}(\sigma-a)^{k} d \sigma\right. \\
\left.+\left(\frac{t-a}{b-a}\right)^{k} \int_{t}^{b}(b-\sigma)^{k} d \sigma\right]^{1 / k} & =\frac{(b-t)(t-a)}{(b-a)^{1-1 / k}(k+1)^{1 / k}} .
\end{aligned}
$$

The function $t \rightarrow(b-t)(t-a), t \in[a, b]$, takes its maximum for $t=(a+b) / 2$. Therefore,

$$
\left(\int_{a}^{b}|G(t, \sigma)|^{k} d \sigma\right)^{1 / k} \leqslant \frac{(b-a)^{1+1 / k}}{4(k+1)^{1 / k}}
$$

for every $t \in[a, b]$. This shows $\left(i_{1}\right)$. The proof of $\left(i_{2}\right)$ is similar to that of $\left(i_{1}\right)$; hence we omit it. Since $\left(i_{3}\right)$ is well-known (see, for instance, $\left.[6], p .422\right)$ and $\left(i_{4}\right)$ is trivial, our claim is proved.

\section{Results}

Let $\|\cdot\|_{1},\|\cdot\|_{2},\|\cdot\|_{3}$ be three fixed norms on $\mathbb{R}^{n} ; d_{3}$ the metric induced by $\|\cdot\|_{3}$; $\|\cdot\|_{\mathbb{R}^{n} \times \mathbb{R}^{n}}$ the norm on $\mathbb{R}^{n} \times \mathbb{R}^{n}$ defined by putting, for every $(x, z) \in \mathbb{R}^{n} \times \mathbb{R}^{n}$,

$$
\|(x, z)\|_{\mathbb{R}^{n} \times \mathbb{R}^{n}}= \begin{cases}\max \left\{\frac{4}{b-a}\|x\|_{1},\|z\|_{2}\right\} & \text { if } b-a \leqslant 4 \\ \max \left\{\|x\|_{1}, \frac{b-a}{4}\|z\|_{2}\right\} & \text { if } b-a>4 .\end{cases}
$$

If $C_{1}, C_{2}$ are two positive constants such that

$$
\|x\|_{1} \leqslant C_{1}\|x\|_{3}, \quad\|x\|_{2} \leqslant C_{2}\|x\|_{3}
$$


for every $x \in \mathbb{R}^{n}$, put

$$
\gamma=\max \left\{C_{1}, C_{2}\right\} \gamma^{\prime}
$$

where

$$
\gamma^{\prime}= \begin{cases}1 & \text { if } p=1 \\ {\left[\frac{(b-a)(p-1)}{2 p-1}\right]^{1-1 / p}} & \text { if } 1<p<+\infty \\ (b-a) / 2 & \text { if } p=+\infty\end{cases}
$$

or

$$
\gamma^{\prime}= \begin{cases}(b-a) / 4 & \text { if } p=1 \\ \frac{b-a}{4}\left[\frac{(b-a)(p-1)}{2 p-1}\right]^{1-1 / p} & \text { if } 1<p<+\infty \\ (b-a)^{2} / 8 & \text { if } p=+\infty,\end{cases}
$$

according to whether $b-a \leqslant 4$ or $b-a>4$. Our main result is the following

THEOREM 2.1. Let $F:[a, b] \times \mathbb{R}^{n} \times \mathbb{R}^{n} \rightarrow 2^{\mathbb{R}^{n}}$ be a multifunction with nonempty closed convex values. Assume that:

(i) for almost every $t \in[a, b]$ the multifunction $F(t, \cdot, \cdot)$ has closed graph;

(ii) the set $\left\{(x, z) \in \mathbb{R}^{n} \times \mathbb{R}^{n}\right.$ : the multifunction $F(\cdot, x, z)$ is measurable $\}$ is dense in $\mathbb{R}^{n} \times \mathbb{R}^{n}$;

(iii) there exist $p, s \in[1,+\infty]$, with $p \leqslant s$, and $r \in] 0,+\infty[$ such that the function

$t \rightarrow \sup \left\{d_{3}(0, F(t, x, z)):(x, z) \in \mathbb{R}^{n} \times \mathbb{R}^{n},\|(x, z)\|_{\mathbb{R}^{n} \times \mathbb{R}^{n}} \leqslant \gamma r\right\}$

belongs to $L^{\prime}([a, b], \mathbb{R})$ and its norm in $L^{p}([a, b], \mathbb{R})$ is less than or equal to $r$.

Then, problem (P) has at least one generalised solution $u \in W^{2, \imath}\left([a, b], \mathbb{R}^{n}\right)$. Moreover, for almost every $t \in[a, b]$, one has

$$
\left\|u^{\prime \prime}(t)\right\|_{3} \leqslant \sup \left\{d_{3}(0, F(t, x, z)):(x, z) \in \mathbb{R}^{n} \times \mathbb{R}^{n},\|(x, z)\|_{\mathbb{R}^{n} \times \mathbb{R}^{n}} \leqslant \gamma r\right\} .
$$

Proof: Let us apply Theorem 1 of [7]. To this end, choose: $T=[a, b]$ with the Lebesgue measure structure; $V=\left\{u \in W^{2, s}\left([a, b], \mathbb{R}^{n}\right): u(a)=u(b)=0\right\}$; $\left(X,\|\cdot\|_{X}\right)=\left(\mathbb{R}^{n} \times \mathbb{R}^{n},\|\cdot\|_{\mathbb{B}^{n} \times \mathbb{R}^{n}}\right) ;\left(Y,\|\cdot\|_{Y}\right)=\left(\mathbb{R}^{n},\|\cdot\|_{s}\right) ; q=1$ and $p, s, r$ such as in (iii); $\Psi(u)=u^{\prime \prime}$ for all $u \in V ; \Phi(u)(t)=\left(u(t), u^{\prime}(t)\right) \in \mathbb{R}^{n} \times \mathbb{R}^{n}$ for all $u \in V$, $t \in[a, b] ; \varphi(\lambda)=\gamma \lambda$ for all $\lambda \in[0,+\infty[$, where $\gamma$ is given by (2). It is easy to check 
that $\Psi$ is a one-to-one operator from $V$ onto $L^{a}([a, b], Y)$. Moreover, thanks to (1), for every $w \in L^{s}([a, b], Y)$ and every $t \in[a, b]$, one has:

$$
\Phi\left(\Psi^{-1}(w)\right)(t)=\left(-\int_{a}^{b} G(t, \sigma) w(\sigma) d \sigma,-\int_{a}^{b} \frac{\partial G(t, \sigma)}{\partial t} w(\sigma) d \sigma\right) .
$$

Now, let $v \in L^{\bullet}([a, b], Y)$ and let $\left\{v_{k}\right\}$ be a sequence in $L^{\bullet}([a, b], Y)$ weakly converging to $v$ in $L^{1}([a, b], Y)$. Since $G(t, \cdot), \partial G(t, \cdot) / \partial t \in L^{\infty}([a, b], \mathbb{R})$ for all $t \in[a, b]$, from (5) it follows that the sequence $\left\{\Phi\left(\Psi^{-1}\left(v_{k}\right)\right)\right\}$ converges pointwise to $\Phi\left(\Psi^{-1}(v)\right)$ on $[a, b]$. Taking into account that $\left\{v_{k}\right\}$ is bounded in $L^{1}([a, b], Y)$ and that, by Lemma 1.1, for every $t \in[a, b]$ and every $k \in \mathbb{N}$ one has

$$
\begin{gathered}
\left\|\int_{a}^{b} G(t, \sigma) v_{k}(\sigma) d \sigma\right\|_{3} \leqslant \frac{b-a}{4} \int_{a}^{b}\left\|v_{k}(\sigma)\right\|_{3} d \sigma, \\
\left\|\int_{a}^{b} \frac{\partial G(t, \sigma)}{\partial t} v_{k}(\sigma) d \sigma\right\|_{3} \leqslant \int_{a}^{b}\left\|v_{k}(\sigma)\right\|_{3} d \sigma,
\end{gathered}
$$

we find that the sequence $\left\{\Phi\left(\Psi^{-1}\left(v_{k}\right)\right)\right\}$ is bounded in $L^{\infty}([a, b], X)$. Hence, by the Lebesgue dominated convergence theorem, $\left\{\Phi\left(\Psi^{-1}\left(v_{k}\right)\right)\right\}$ converges strongly to $\Phi\left(\Psi^{-1}(v)\right)$ in $L^{1}([a, b], X)$.

Next, we prove that for every $u \in V$ one has

$$
\underset{t \in[a, b]}{\operatorname{esssup}}\|\Phi(u)(t)\|_{X} \leqslant \varphi\left(\|\Phi(u)\|_{L^{p}([a, b], Y)}\right) .
$$

We verify this only for $b-a \leqslant 4$ and $p \in] 1,+\infty[$, since in the other cases the proof is similar. To this end, fix $u \in V$ and $t \in[a, b]$. Thanks to Lemma 1.1, we get:

$$
\begin{aligned}
\|u(t)\|_{1} & \leqslant\left(\int_{a}^{b}|G(t, \sigma)|^{p /(p-1)} d \sigma\right)^{1-1 / p}\left(\int_{a}^{b}\left\|u^{\prime \prime}(\sigma)\right\|_{1}^{p} d \sigma\right)^{1 / p} \\
& \leqslant \frac{b-a}{4}\left[\frac{(b-a)(p-1)}{2 p-1}\right]^{1-1 / p} C_{1}\|\Psi(u)\|_{L^{p}([a, b], Y)} \\
\left\|u^{\prime}(t)\right\|_{2} & \leqslant\left(\int_{a}^{b}\left|\frac{\partial G(t, \sigma)}{\partial t}\right|^{p /(p-1)} d \sigma\right)^{1-1 / p}\left(\int_{a}^{b}\left\|u^{\prime \prime}(\sigma)\right\|_{2}^{p} d \sigma\right)^{1 / p} \\
& \leqslant\left[\frac{(b-a)(p-1)}{2 p-1}\right]^{1-1 / p} C_{2}\|\Psi(u)\|_{L^{p}([a, b], Y)} .
\end{aligned}
$$

This implies that

$$
\begin{aligned}
\max & \left\{\frac{4}{b-a}\|u(t)\|_{1},\left\|u^{\prime}(t)\right\|_{2}\right\} \\
& \leqslant \max \left\{C_{1}, C_{2}\right\}\left[\frac{(b-a)(p-1)}{2 p-1}\right]^{1-1 / p}\|\Psi(u)\|_{L^{p}([a, b], Y)}
\end{aligned}
$$


for every $t \in[a, b]$. Hence

$$
\begin{aligned}
& \underset{t \in[a, b]}{\operatorname{ess} \sup _{\mathbf{p}}}\|\Phi(u)(t)\|_{X} \\
& \quad \leqslant \max \left\{C_{1}, C_{2}\right\}\left[\frac{(b-a)(p-1)}{2 p-1}\right]^{1-1 / p}\|\Psi(u)\|_{L^{P}([a, b], Y)} .
\end{aligned}
$$

At this point, we are able to apply Theorem 1 of [7]. By that result, there exists $u \in V$ such that

$$
u^{\prime \prime}(t) \in F\left(t, u(t), u^{\prime}(t)\right)
$$

almost everywhere in $[a, b]$ and

$$
\left\|u^{\prime \prime}(t)\right\|_{3} \leqslant \sup \left\{d_{3}(0, F(t, x, z)):(x, z) \in \mathbb{R}^{n} \times \mathbb{R}^{n},\|(x, z)\|_{\mathbb{R}^{n} \times \mathbb{R}^{n}} \leqslant \gamma r\right\}
$$

almost everywhere in $[a, b]$. This completes the proof.

From now on, $\|\cdot\|_{\mathbb{R}^{n}}$ will denote a fixed norm on $\mathbb{R}^{n}$ and $d$ the metric induced by $\|\cdot\|_{\mathbb{R}^{n}}$. If $\|\cdot\|_{1}=\|\cdot\|_{2}=\|\cdot\|_{3}=\|\cdot\|_{\mathbb{R}^{n}}$ and $p=s$, Theorem 2.1 assumes the following form, which improves Theorem 3 of [7].

TheOREM 2.2. Let $F$ satisfy assumptions (i) and (ii) of Theorem 2.1. Further, suppose that:

(j) there exist $p \in[1,+\infty]$ and $r \in] 0,+\infty[$ such that

$$
\begin{aligned}
& \underset{t \in[a, b]}{\operatorname{ess} \sup \sup }\left\{d(0, F(t, x, z)):\|x\|_{\mathbb{R}^{n}} \leqslant \frac{(b-a)^{2}}{8} r,\|z\|_{\mathbb{R}^{n}} \leqslant \frac{b-a}{2} r\right\} \leqslant r \\
& \text { if } p=+\infty \text {, } \\
& \left(\int_{a}^{b}\left(\sup \left\{d(0, F(t, x, z)):\|x\|_{\mathbb{R}^{n}} \leqslant \gamma_{1} \gamma^{\prime} r,\|z\|_{\mathbb{R}^{n}} \leqslant \gamma_{2} \gamma^{\prime} r\right\}\right)^{p} d t\right)^{1 / p} \leqslant r \\
& \text { if } p \in[1,+\infty[\text {, }
\end{aligned}
$$

where

$$
\gamma_{1}=\left\{\begin{array}{ll}
(b-a) / 4 & \text { if } b-a \leqslant 4 \\
1 & \text { if } b-a>4
\end{array}, \quad \gamma_{2}= \begin{cases}1 & \text { if } b-a \leqslant 4 \\
4 /(b-a) & \text { if } b-a>4\end{cases}\right.
$$

and $\gamma^{\prime}$ is given by (3) or (4), according to whether $b-a \leqslant 4$ or $b-a>4$. Then, problem $(P)$ has at least one generalised solution $u \in W^{2, p}\left([a, b], \mathbb{R}^{n}\right)$. Moreover, for almost every $t \in[a, b]$, one has

$$
\left\|u^{\prime \prime}(t)\right\|_{\mathbb{R}^{n}} \leqslant \sup \left\{d(0, F(t, x, z)):\|x\|_{\mathbb{R}^{n}} \leqslant \gamma_{1} \gamma^{\prime} r,\|z\|_{\mathbb{R}^{n}} \leqslant \gamma_{2} \gamma^{\prime} r\right\} .
$$

When $F$ is single-valued and continuous, Theorem 2.2 assumes the following form (see Theorem 4.2, p.424, of [6]). 
THEOREM 2.3. Let $f$ be a continuous function from $[a, b] \times \mathbb{R}^{n} \times \mathbb{R}^{n}$ into $\mathbb{R}^{n}$. Suppose that there exists $r \in] 0,+\infty[$ such that

$$
\|f(t, x, z)\|_{\mathbb{R}^{n}} \leqslant r
$$

for every $t \in[a, b]$ and every $x, z \in \mathbb{R}^{n}$ such that $\|x\|_{\mathbb{R}^{n}} \leqslant\left((b-a)^{2} / 8\right) r,\|z\|_{\mathbb{R}^{n}} \leqslant$ $((b-a) / 2) r$.

Then, there exists $u \in C^{2}\left([a, b], \mathbb{R}^{n}\right)$ such that

$$
\begin{gathered}
u^{\prime \prime}(t)=f\left(t, u(t), u^{\prime}(t)\right) \text { for every } t \in[a, b] \\
u(a)=u(b)=0, \\
\|u(t)\|_{\mathbb{R}^{n}} \leqslant \frac{(b-a)^{2}}{8} r,\left\|u^{\prime}(t)\right\|_{\mathbb{R}^{n}} \leqslant \frac{b-a}{2} r \text { for every } t \in[a, b] .
\end{gathered}
$$

Proof: Thanks to our assumptions, we are allowed to apply Theorem 2.2, with $p=+\infty$, to the multifunction $F$ defined by putting, for every $(t, x, z) \in[a, b] \times \mathbb{R}^{n} \times$ $\mathbb{R}^{n}, F(t, x, z)=\{f(t, x, z)\}$. There is, therefore, $u_{1} \in W^{2, \infty}\left([a, b], \mathbb{R}^{n}\right)$ such that $u_{1}(a)=u_{1}(b)=0$ and

$$
\begin{gathered}
u_{1}^{\prime \prime}(t)=f\left(t, u_{1}(t), u_{1}^{\prime}(t)\right) \\
\left\|u_{1}^{\prime \prime}(t)\right\|_{\mathbb{R}^{n}} \leqslant r
\end{gathered}
$$

for almost every $t \in[a, b]$. Taking into account that the function $t \rightarrow f\left(t, u_{1}(t), u_{1}^{\prime}(t)\right)$ is continuous, from (6) it follows that there exists $u \in C^{2}\left([a, b], \mathbb{R}^{n}\right)$ such that $u^{\prime \prime}(t)=$ $u_{1}^{\prime \prime}(t)$ almost everywhere in $[a, b], u(a)=u(b)=0, u^{\prime \prime}(t)=f\left(t, u(t), u^{\prime}(t)\right)$ for every $t \in[a, b]$. Thanks to (1) and (7), this implies that

$$
\|u(t)\|_{\mathbb{R}^{n}} \leqslant r \int_{a}^{b}|G(t, \sigma)| d \sigma,\left\|u^{\prime}(t)\right\|_{\mathbb{R}^{n}} \leqslant r \int_{a}^{b}\left|\frac{\partial G(t, \sigma)}{\partial t}\right| d \sigma
$$

for every $t \in[a, b]$. Hence, by Lemma 1.1,

$$
\|u(t)\|_{\mathbb{R}^{n}} \leqslant \frac{(b-a)^{2}}{8} r, \quad\left\|u^{\prime}(t)\right\|_{\mathbb{R}^{n}} \leqslant \frac{b-a}{2} r
$$

for all $t \in[a, b]$.

REMARK 2.1. A simple sufficient condition in order that (j) of Theorem 2.2 holds is the following.

(j) There exist $p \in[1,+\infty]$ and $\alpha, \beta, \chi \in L^{p}([a, b], \mathbb{R})$ such that

$$
\gamma_{1} \gamma^{\prime}\|\alpha\|_{L^{P}([a, b], \mathbf{1})}+\gamma_{2} \gamma^{\prime}\|\beta\|_{L P([a, b], \mathbf{3})}<1
$$


(where $\gamma_{1}, \gamma_{2}, \gamma^{\prime}$ are such as in Theorem 2.2 and $\|\cdot\|_{L^{p}([a, b], \mathbb{B})}$ is the usual norm of $\left.L^{p}([a, b], \mathbb{R})\right)$ and

$$
d(0, F(t, x, z)) \leqslant \alpha(t)\|x\|_{\mathbb{R}^{n}}+\beta(t)\|z\|_{\mathbb{I}^{n}}+\chi(t)
$$

for almost every $t \in[a, b]$ and every $x, z \in \mathbb{R}^{n}$.

To verify this, it suffices to choose

$$
r>\frac{\|\chi\|_{L^{p}([a, b], \mathbb{R})}}{1-\left(\gamma_{1} \gamma^{\prime}\|\alpha\|_{L^{p}([a, b], \mathbb{R})}+\gamma_{2} \gamma^{\prime}\|\beta\|_{L^{p}([a, b], \mathbb{E})}\right)}
$$

and to observe that, thanks to (8), one has:

$$
\begin{aligned}
& \left(\int_{a}^{b}\left(\sup \left\{d(0, F(t, x, z)):\|x\|_{\mathbb{R}^{n}} \leqslant \gamma_{1} \gamma^{\prime} r,\|z\|_{\mathbb{R}^{n}} \leqslant \gamma_{2} \gamma^{\prime} r\right\}\right)^{p} d t\right)^{1 / p} \\
& \leqslant\left[\gamma_{1} \gamma^{\prime}\|\alpha\|_{L^{P}([a, b], \mathbb{R})}+\gamma_{2} \gamma^{\prime}\|\beta\|_{L^{P}([a, b], \mathbb{R})}\right] r+\|\chi\|_{L^{p}([a, b], \mathbb{R})} \\
& \text { for } p \in[1,+\infty[\text {; } \\
& \underset{t \in[a, b]}{\text { ess sup sup }}\left\{d(0, F(t, x, z)):\|x\|_{\mathbb{R}^{n}} \leqslant \frac{(b-a)^{2}}{8} r,\|z\|_{\mathbb{R}^{n}} \leqslant \frac{b-a}{2} r\right\} \\
& \leqslant\left[\frac{(b-a)^{2}}{8}\|\alpha\|_{L^{\infty}([a, b], \mathbb{R})}+\frac{b-a}{2}\|\beta\|_{L^{\infty}([a, b], \mathbb{R})}\right] r+\|\chi\|_{L^{\infty}([a, b], \mathbb{R})} \\
& \text { for } p=+\infty \text {. }
\end{aligned}
$$

It is worth noticing that Theorem 2.2, with (jj) instead of ( $\mathrm{j}$ ), improves and extends to multi-valued boundary value problems some classical results, such as the Theorem of p.256 of [9] and Theorem III of [2].

For other existence results for problem $(\mathrm{P})$ where one assumes that $F$ satisfies a growth condition like (8), we refer to Theorem 4.6, p.36, of [8]. It is easy to check that this result and Theorem 2.2 (with (jj) instead of (j)) are mutually independent.

REMARK 2.2. Another simple sufficient condition in order that (j) of Theorem 2.2 holds is the following.

(jij) There exist $p \in[1,+\infty], r \in] 0,+\infty\left[\right.$ and $\alpha(t, x), \beta(t, x):[a, b] \times \mathbb{R}^{n} \rightarrow$ $\mathbb{R}$, measurable with respect to $t$ for every fixed $x \in \mathbb{R}^{n}$ and continuous with respect to $x$ for every fixed $t \in[a, b]$, such that:

for almost every $t \in[a, b]$ and every $x, z \in \mathbb{R}^{n}$ one has

$$
d(0, F(t, x, z)) \leqslant \alpha(t, x)\|z\|_{\mathbb{R}^{n}}^{2}+\beta(t, x) ;
$$


if $\gamma_{1}, \gamma_{2}, \gamma^{\prime}$ are such as in Theorem 2.2, then

$$
\begin{aligned}
& \left(\gamma_{2} \gamma^{\prime}\right)^{2}\left(\int_{a}^{b}\left(\sup \left\{\alpha(t, x):\|x\|_{\mathbb{R}^{n}} \leqslant \gamma_{1} \gamma^{\prime} r\right\}\right)^{p} d t\right)^{1 / p} r^{2} \\
& +\left(\int_{a}^{b}\left(\sup \left\{\beta(t, x):\|x\|_{\mathbb{R}^{n}} \leqslant \gamma_{1} \gamma^{\prime} r\right\}\right)^{p} d t\right)^{1 / p} \leqslant r
\end{aligned}
$$

or

$$
\begin{gathered}
\frac{(b-a)^{2}}{4} \operatorname{ess~sup~sup~}_{t \in[a, b]}\left\{\alpha(t, x):\|x\|_{\mathbb{R}^{n}} \leqslant \frac{(b-a)^{2}}{8} r\right\} r^{2} \\
+\underset{t \in[a, b]}{\operatorname{ess} \sup \sup }\left\{\beta(t, x):\|x\|_{\mathbb{R}^{n}} \leqslant \frac{(b-a)^{2}}{8} r\right\} \leqslant r,
\end{gathered}
$$

according to whether $p \in[1,+\infty[$ or $p=+\infty$.

The proof that $(\mathrm{jij}) \Rightarrow(\mathrm{j})$ is trivial; hence we omit it.

For other existence results for problem (P) where one assumes that $F$ satisfies a growth condition like (9), we refer to Theorem 4 of [5]. It is easy to verify that this result and Theorem 2.2 (with (jij) instead of (j)) are mutually independent.

The next result can be regarded as the multi-valued analogue of Theorem 9 of [10].

Theorem 2.4. Let $F$ satisfy assumptions (i) and (ii) of Theorem 2.1. Further, suppose that there exists $p \in[1,+\infty]$ such that for every $\tau>0$ there is $h_{\tau} \in L^{p}([a, b], \mathbb{R})$ such that

$$
\sup \left\{d(0, F(t, x, z)):\|x\|_{\mathbb{R}^{n}} \leqslant \tau,\|z\|_{\mathbb{R}^{n}} \leqslant \tau\right\} \leqslant h_{\tau}(t)
$$

for almost every $t \in[a, b]$.

Then, for every $\varepsilon>0$ there exists $\delta_{\varepsilon}>0$ such that, if $\left[a_{1}, b_{1}\right] \subseteq[a, b]$ and $b_{1}-a_{1}<\delta_{e}$, then the problem

$$
\left\{\begin{array}{l}
u^{\prime \prime} \in F\left(t, u, u^{\prime}\right) \\
u\left(a_{1}\right)=u\left(b_{1}\right)=0
\end{array}\right.
$$

has at least one generalised solution $u \in W^{2}, p\left(\left[a_{1}, b_{1}\right], \mathbb{R}^{n}\right)$. Moreover, for every $t \in$ $\left[a_{1}, b_{1}\right]$, one has

$$
\|u(t)\|_{\mathbb{R}^{n}} \leqslant \varepsilon
$$

Proof: Fix $\varepsilon>0$ and choose

$$
\tau=\varepsilon \max \left\{1, \frac{4}{b-a}\right\}
$$


Taking into account that $h_{\tau} \in L^{p}([a, b], \mathbb{R})$, we get $\delta_{\varepsilon}>0$ such that, if $\left[a_{1}, b_{1}\right] \subseteq$ $[a, b]$ and $b_{1}-a_{1}<\delta_{\varepsilon}$, then

$$
\begin{aligned}
& \left(\int_{a_{1}}^{b_{1}}\left|h_{\tau}(t)\right|^{p} d t\right)^{1 / p} \leqslant \frac{\varepsilon}{\gamma_{1} \gamma^{\prime}} \quad \text { for } p \in[1,+\infty[, \\
& \underset{t \in\left[a_{1}, b_{1}\right]}{\operatorname{ess} \sup _{\tau}}\left|h_{\tau}(t)\right| \leqslant \frac{\varepsilon}{\gamma_{1} \gamma^{\prime}} \quad \text { for } p=+\infty \text {, }
\end{aligned}
$$

where $\gamma_{1}$ and $\gamma^{\prime}$ are such as in Theorem 2.2. Now, fix $\left[a_{1}, b_{1}\right] \subseteq[a, b]$ with $b_{1}-a_{1}<\delta_{e}$. Thanks to our assumptions, the multifunction $\left.F\right|_{\left[a_{1}, b_{1}\right] \times \mathbb{R}^{n} \times \mathbb{R}^{n}}$ satisfies (i) and (ii) of Theorem 2.1. Moreover, if we take

$$
r=\frac{\varepsilon}{\gamma_{1} \gamma^{\prime}}
$$

then, from (10), (11) and (12) it follows that

$$
\begin{aligned}
& \left(\int_{a_{1}}^{b_{1}}\left(\sup \left\{d(0, F(t, x, z)):\|x\|_{\mathbb{R}^{n}} \leqslant \gamma_{1} \gamma^{\prime} r,\|z\|_{\mathbb{R}^{n}} \leqslant \gamma_{2} \gamma^{\prime} r\right\}\right)^{p} d t\right)^{1 / p} \\
& \leqslant\left(\int_{a_{1}}^{b_{1}}\left(\sup \left\{d(0, F(t, x, z)):\|x\|_{\mathbb{R}^{n}} \leqslant \tau,\|z\|_{\mathbb{R}^{n}} \leqslant \tau\right\}\right)^{p} d t\right)^{1 / p} \leqslant r \\
& \quad \text { for } p \in[1,+\infty[, \\
& \text { ess sup sup }\left\{d(0, F(t, x, z)):\|x\|_{\mathbb{R}^{n}} \leqslant \frac{(b-a)^{2}}{8} r,\|z\|_{\mathbb{R}^{n}} \leqslant \frac{b-a}{2} r\right\} \leqslant r \\
& \begin{array}{c}
t \in\left[a_{1}, b_{1}\right] \\
\text { for } p=+\infty .
\end{array}
\end{aligned}
$$

At this point, we are allowed to apply Theorem 2.2 to the multifunction $\left.F\right|_{\left[a_{1}, b_{1}\right] \times \mathbb{R}^{n} \times \mathbb{R}^{n}}$. There is, therefore, a generalised solution $u \in W^{2, p}\left(\left[a_{1}, b_{1}\right], \mathbb{R}^{n}\right)$ of the problem $\left(P_{1}\right)$ such that

$$
\left\|u^{\prime \prime}(t)\right\|_{\mathbb{B}^{n}} \leqslant h_{\tau}(t)
$$

for almost every $t \in[a, b]$. Next, observe that, by (1), (12) and Lemma 1.1, for every $t \in\left[a_{1}, b_{1}\right]$ one has:

$$
\begin{aligned}
& \|u(t)\|_{\mathbb{R}^{n}} \leqslant \operatorname{esssup}_{\sigma \in[a, b]}|G(t, \sigma)| \int_{a_{1}}^{b_{1}}\left|h_{\tau}(t)\right| d t \leqslant \frac{b-a}{4} \frac{\varepsilon}{(b-a) / 4}=\varepsilon \quad \text { for } \quad p=1 \\
& \|u(t)\|_{\mathbb{R}^{n}} \leqslant\left(\int_{a}^{b}|G(t, \sigma)|^{p /(p-1)} d \sigma\right)^{1-1 / p}\left(\int_{a_{1}}^{b_{1}}\left|h_{\tau}(t)\right|^{p} d t\right)^{1 / p} \leqslant \gamma_{1} \gamma^{\prime} \frac{\varepsilon}{\gamma_{1} \gamma^{\prime}}=\varepsilon \\
& \text { for } p \in] 1,+\infty[\text {; } \\
& \|u(t)\|_{\mathbb{R}^{n}} \leqslant \int_{a}^{b}|G(t, \sigma)| d \sigma \text { ess sup } \sin _{t \in\left[a_{1}, b_{1}\right]}\left|h_{\tau}(t)\right| \leqslant \frac{(b-a)^{2}}{8} \frac{\varepsilon}{(b-a)^{2} / 8}=\varepsilon \text { for } p=+\infty \text {. }
\end{aligned}
$$


Hence, in any case,

$$
\|u(t)\|_{\mathbb{B}^{n}} \leqslant \varepsilon
$$

for every $t \in\left[a_{1}, b_{1}\right]$. This completes the proof.

Finally, we give a very simple example of an application of Theorem 2.2, where it is impossible to apply any of the theorems just now quoted.

EXAMPLE 2.1. Let $p \in[1,+\infty]$ and let $\varphi \in L^{p}([a, b], \mathbb{R})$ such that

$$
\|\varphi\|_{L^{p}([a, b], \mathbb{R})} \leqslant \frac{1}{\gamma^{\prime} e}
$$

where $\gamma^{\prime}$ is given by (3) or (4), according to whether $b-a \leqslant 4$ or $b-a>4$.

Then, the problem

$$
\left\{\begin{array}{l}
u^{\prime \prime}=\varphi(t) e^{\max \left\{|u|,\left|u^{\prime}\right|\right\}} \\
u(a)=u(b)=0
\end{array}\right.
$$

has at least one generalised solution $u \in W^{2, p}([a, b], \mathbb{R})$. Moreover, for almost every $t \in[a, b]$, one has

$$
\left|u^{\prime \prime}(t)\right| \leqslant e|\varphi(t)| .
$$

Proof: For every $(t, x, z) \in[a, b] \times \mathbb{R} \times \mathbb{R}$, put:

$$
F(t, x, z)=\left\{\varphi(t) e^{\max \{|x|,|z|\}}\right\} .
$$

Of course, the multifunction $F:[a, b] \times \mathbb{R} \times \mathbb{R} \rightarrow 2^{\mathbb{R}}$ so defined satisfies assumptions (i) and (ii) of Theorem 2.1. Moreover, if we take $r=1 / \gamma^{\prime}$, thanks to (13) one has:

$$
\begin{aligned}
& \left(\int_{a}^{b}\left(\sup \left\{|\varphi(t)| e^{\max \{|x|,|z|\}}:|x| \leqslant \gamma_{1},|z| \leqslant \gamma_{2}\right\}\right)^{p} d t\right)^{1 / p} \\
& \quad=e^{\max \left\{\gamma_{1}, \gamma_{2}\right\}}\|\varphi\|_{L^{p}([a, b], \mathbb{R})} \leqslant \frac{1}{\gamma^{\prime}} \quad \text { for } p \in[1,+\infty[; \\
& \underset{t \in[a, b]}{\text { ess sup sup}\left\{|\varphi(t)| e^{\max \{|x|,|z|\}}:|x| \leqslant \gamma_{1},|z| \leqslant \gamma_{2}\right\}} \\
& \quad=e^{\max \left\{\gamma_{1}, \gamma_{2}\right\}}\|\varphi\|_{L^{\infty}([a, b], \mathbb{R})} \leqslant \frac{1}{\gamma^{\prime}} \quad \text { for } p=+\infty .
\end{aligned}
$$

This shows that (j) of Theorem 2.2 holds. Hence, by that result, there is $u \in$ $W^{2, p}([a, b], \mathbb{R})$ such that $u^{\prime \prime}(t)=\varphi(t) e^{\max \left\{|u(t)|,\left|u^{\prime}(t)\right|\right\}}$ almost everywhere in $[a, b]$, $u(a)=u(b)=0$ and $\left|u^{\prime \prime}(t)\right| \leqslant e|\varphi(t)|$ for almost every $t \in[a, b]$.

It is worth noticing that Theorem 3 of [7] does not apply to the previous problem when, for instance, $b-a>1$ and $\|\varphi\|_{L^{p}([a, b], \mathbb{1})}>1 /\left((b-a)^{2-1 / p} e\right)$. 


\section{REFERENCES}

[1] S.R. Bernfeld and V. Lakshmikantham, An introduction to nonlinear boundary value problems (Academic Press, New York, London, 1974).

[2] S. Cinquini, 'Problemi di valori al contorno per equazioni differenziali (non lineari) del secondo ordine', Ann. Scuola Norm. Sup. Pisa Cl. Sci. (2) 8 (1939), 1-22.

[3] L.H. Erbe and W. Krawcewicz, 'Boundary value problems for differential inclusions', in Lecture Notes in Pure and Appl. Math. 127, pp. 115-135 (Marcel Dekker, 1991).

[4] M. Frigon and A. Granas, 'Théorèmes d'existence pour des inclusions différentielles sans convexité', C.R. Acad. Sci. Paris, Série I Math. 310 (1990), 819-822.

[5] A. Granas, R.B. Guenther and J.W. Lee, 'Some existence results for the differential inclusions $y^{(k)} \in F\left(x, y, \ldots, y^{(k-1)}\right), y \in \mathcal{B}^{\prime}$, C.R. Acad. Sci. Paris, Série I Math. 307 (1988), 391-396.

[6] P. Hartman, Ordinary differential equations (John Wiley and Sons, New York, London, Sydney, 1964).

[7] O. Naselli Ricceri and B. Ricceri, 'An existence theorem for inclusions of the type $\Psi(u)(t) \in F(t, \Phi(u)(t))$ and application to a multivalued boundary value problem', Appl. Anal. 38 (1990), 259-270.

[8] T. Pruszko, 'Some applications of the topological degree theory to multi-valued boundary value problems', in Dissertationes Math. 229, pp. 1-48, 1984.

[9] G. Scorza Dragoni, 'Elementi uniti di trasformazioni funzionali e problemi di valori ai limiti', Rend. Sem. Mat. Univ. Roma (4) 2 (1938), 255-275.

[10] H.B. Thompson, 'Minimal solutions for two point boundary value problems', Rend. Circ. Mat. Palermo (2) 37 (1988), 261-281.

Dipartimento di Matematica

Città Universitaria

Viale A. Doria, 6 - 95125

Catania

Italy 